\title{
Towards the simulations of inertial dense particulate flows with a volume-averaged lattice Boltzmann method
}

\author{
Stephan B. Höcker ${ }^{\mathrm{a}, \mathrm{b}}$, Robin Trunk ${ }^{\mathrm{a}, \mathrm{b}}$, Willy Dörfler ${ }^{\mathrm{b}}$, Mathias J. Krause ${ }^{\mathrm{a}, \mathrm{b}, \mathrm{c}, *}$ \\ a Lattice Boltzmann Research Group, Germany \\ ${ }^{\mathrm{b}}$ Institute for Applied and Numerical Mathematics, Germany \\ ${ }^{c}$ Institute for Mechanical Process Engineering and Mechanics, Karlsruhe Institute of Technology, Karlsruhe, Germany
}

Keywords:

CFD

VANS

Dense particulate flows

Backcoupling

Two-way coupling

Euler-Euler

LBM

OpenLB

\begin{abstract}
A B S T R A C T
The simulation of dense particulate flows offers a series of challenges, including the modelling of particleparticle and fluid-particle interaction, complex heterogeneous structures in the particulate phase and the displacement of fluid by particles. A common approach to incorporate the latter into the governing equations of the fluid phase is given by the volume-averaged Navier-Stokes (VANS) equations which have been extensively researched in combination with finite volume methods. Multiple lattice Boltzmann (LB) schemes for the VANS equations have been suggested, yet only one study, relying on the use of non-physical force terms, investigated the schemes' applicability to test cases with non-homogeneous particle concentrations. Furthermore, no such scheme has yet been used in a dense Euler-Euler model.

In this paper, we first introduce a novel lattice Boltzmann method (LBM) for the VANS equations which relies on an adaptation of the streaming step, while requiring no additional forcing terms. Second, we combine the method with an advection-diffusion LBM to obtain a simple multiphase model, which forms a first step towards an Euler-Euler model for dense particulate flows. It takes the phases' volume fractions and simple drag forces into consideration but neglects some inter-particle and hydrodynamic forces as well as turbulence. The LBM's convergence to the VANS equations is investigated in four test cases with analytical solutions, two of which contain spatially or temporally fluctuating particle concentrations. The combined multiphase model is validated using a Rayleigh-Taylor instability test case.
\end{abstract}

\section{Introduction}

Dense particulate flows can be found in many scientific and industrial applications, such as deep bed filters $[1,2]$ or fluidized bed reactors [3]. Accordingly, the correct modelling and efficient simulation of dense particulate flows is of considerable importance. Since they characteristically contain strong interaction between particles as well as strong hydrodynamic interaction $[4,5]$, this is a challenging task.

Due to the substantial presence of particles, simulations which resolve the motion of single particles (Lagrangian representation) are overly computationally expensive [6]. Eulerean models, in which particles are represented by a concentration or density, provide a computationally cheap alternative. The advection-diffusion model in particular includes particle-interaction through a diffu-

\footnotetext{
* Corresponding author at : Institute for Mechanical Process Engineering and Mechanics, Karlsruhe Institute of Technology, Karlsruhe, Germany.

E-mail address: mathias.krause@kit.edu (M.J. Krause).
}

sion term while forces can be included through an adaptation of the bulk-velocity. Since the positions of particles are not resolved in Eulerean representations, a fluid model is needed which implicitly accounts for particle presence and in which the particlefluid interaction can be accurately modelled. A common approach to achieve this is through the procedure of volume-averaging, pioneered by Anderson and Jackson [7]. Using this procedure, a set of equations for the fluid phase can be derived, which inherently contain the interaction with particles [8-10]. The resulting volume-averaged Navier-Stokes (VANS) equations are extensively used in different multiphase models - they for example form the basis of the two-fluid model, but can also be used to simulate the fluid phase for discrete element methods (DEM) and multiphase particle-in-cell (MP-PIC) methods [11,12].

For conventional CFD methods, especially FVM, volumeaveraged models are well established [11]. For lattice Boltzmann methods (LBM) [13,14], whose importance has steadily increased in recent years, no comprehensive implementation has yet been established. The great advantage of the LBMs lies in their scalability, 
which makes them particularly well suited for implementation on high performance computers $[15,16]$. They are based on the Boltzmann equation, a fundamental equation of statistical mechanics and thermodynamics, and therefore rely on a mesoscopic density function. As a result, macroscopic quantities such as momentum, density and pressure are not directly used. The convergence of the methods towards the macroscopic partial differential equations is generally proven through Chapman-Enskog analysis [13]. For the Navier-Stokes equations the procedure is well established whereas proving convergence for the VANS equations is more challenging. Multiple LB schemes for the VANS equations have been suggested, for example those of Guo and Zhao [17], Zhang et al. [18] and Song et al. [19], but as far as the authors of this paper are aware, none of these schemes have yet been used for an Euler-Euler LBM for dense particulate flows. Furthermore, for none of these schemes grid convergence in the case of a non-uniform porosity has been demonstrated, while porosity gradients even occur in simple particulate flows such as sedimentations. Accordingly, for a broader applicability for multiphase flows, a scheme which handles large porosity gradients is required. To the knowledge of the authors, the only paper investigating analytical and numerical convergence for such cases was provided by Blais et al. [12], who introduced a scheme that requires multiple additional forcing terms to retrieve the correct VANS equations.

The aim of this paper is to establish a stable Euler-Euler LBM for multiphase flows, which accurately incorporates a two-way coupling between fluid and particles. This includes the introduction of a novel VANS LBM, which relies on the manipulation of the streaming step and is required to handle both spatially and temporally fluctuating particle concentrations, without the use of nonphysical forcing terms.

The Euler-Euler VANS LBM is expected to form a first step towards a model for dense particulate flows, however some effects are not included in this work. We make the following assumptions: The fluid is incompressible and the hydrodynamic interaction is restricted to a quadratic drag-force. Turbulence, subgrid-stresses and the subsequent effective viscosity are not considered. The particulate phase is assumed to be of uniform size and density while the particle-interaction is modelled through diffusion. Seeing as the diffusion coefficient is chosen to be constant, the close-packing limit is not considered. While these assumption make the EulerEuler VANS LBM, as presented here, inadequate for dense particulate flow, the simple equations for the fluid enable test-cases with analytical solutions and allow for an assessment of the schemes stability in test-cases with high porosity gradients. Seeing as the fluid's forces are simply implemented using a scheme by Guo et al. [20], an extension of the hydrodynamic forces should prove to be rather straightforward.

A short introduction to the VANS equations, the advectiondiffusion equation and coupling-approaches is provided in Section 2. The standard LBM, VANS LBM, and advection-diffusion LBM will be introduced in Section 3, followed by some remarks on the implementation of the phase-coupling. Here, Guo and Zhao's forcing scheme for drag forces [17] is extended to non-static particles. The coupled Euler-Euler VANS LBM is then introduced in Section 3.5 Grid-convergence for VANS LBM to an analytical solution is investigated for two test cases with a uniform, static particle phase, as well as two test cases with spatially and temporally fluctuating particle-concentrations in Section 4 . Then, the Euler-Euler VANS LBM is validated by simulating a Rayleigh-Taylor instability. In each test case, a standard LBM forcing scheme will be compared to the adapted forcing scheme from Section 3.4. The results will be discussed in Section 5, while the VANS LBM's performance in the hydrodynamic limit is investigated in Appendix A using Chapman-Enskog analysis.

\section{Mathematical modeling}

\subsection{The volume-averaged Navier-Stokes equations}

In order to derive the VANS equations, an averaging operation $\langle\cdot\rangle$ is used, which, for some function $\phi$ at position $\mathbf{x}$, is given by

$\langle\phi\rangle(\mathbf{x})=\frac{\int_{\Omega} \phi(\mathbf{y}) g(\mathbf{x}-\mathbf{y}) \chi(\mathbf{y}) d \mathbf{y}}{\int_{\Omega} g(\mathbf{x}-\mathbf{y}) \chi(\mathbf{y}) d \mathbf{y}}$.

$g: \Omega \rightarrow \mathbb{R}$ is a smoothing kernel which is normalized to yield one when integrated over the entire domain $\Omega \subset \mathbb{R}^{d}$. The phase identification function $\chi: \Omega \rightarrow\{0,1\}$ assumes the value one if $\mathbf{x}$ is occupied by the fluid and zero elsewhere. The denominator $\epsilon(\mathbf{x}):=$ $\int_{\Omega} g(\mathbf{x}-\mathbf{y}) \chi(\mathbf{y}) d \mathbf{y}$ in Eq. (1) represents the fluid's volume fraction in $\mathbf{x}$, which is commonly referred to as porosity or void. Using a suitable averaging operator [21], the new governing equations for the fluid phase can be derived from the Navier-Stokes equations. In these new equations, the bulk-flows are resolved and the fluid-particle interaction is implicitly included through the hydrodynamic force term $\mathbf{F}^{h}$ and a subgrid-stress term $\sigma_{S G}$ which represents particle-induced stresses and turbulence [22]. This subgridstress is neglected in this work, which allows for simpler analytical solutions for the test cases.

The resulting mass- and momentum conservation equations with density $\rho$, velocity $\mathbf{u}$, body force $\mathbf{F}^{f}$ and pressure $p$ at position $\mathbf{x}$ and time $t$ are given by Enwald et al. [8]

$$
\begin{aligned}
& \frac{\partial}{\partial t} \epsilon \rho+\nabla \cdot(\epsilon \rho\langle\mathbf{u}\rangle)=0 \\
& \frac{\partial \epsilon \rho\langle\mathbf{u}\rangle}{\partial t}+\nabla \cdot\left(\epsilon \rho\langle\mathbf{u}\rangle\langle\mathbf{u}\rangle^{\top}\right)+\epsilon \nabla\langle p\rangle \\
& =\mu \Delta(\epsilon\langle\mathbf{u}\rangle)+\epsilon \mathbf{F}^{f}+\mathbf{F}^{h}+\nabla \cdot \sigma_{S G},
\end{aligned}
$$

These equations are called the volume-averaged Navier-Stokes equations. It is clear that Eqs. (2) and (3) still closely resemble the Navier-Stokes equations with a density that is scaled by the porosity; in fact, they are recovered for $\epsilon \rightarrow 1$. This similarity can be utilized for the derivation of a VANS LBM scheme.

It should furthermore be noted, that the initial problem with complicated boundary conditions is transformed into a smoother problem through the usage of the averaging operator, but now includes the terms $\mathbf{F}^{h}$ and $\boldsymbol{\sigma}_{S G}$ which need to be modelled to represent the complex particle-scale behaviour.

\subsection{The advection-diffusion equation}

The particle phase can be modelled by an advection-diffusion model. Particle presence is represented by the quantity $\rho_{p}$, the mass of particles per volume of the flow, which under the assumption of identical particles is proportional to the particle concentration. The advection-diffusion equation, as it is used here, is a mass-conservation equation which includes the dispersion of particles along gradients:

$$
\frac{\partial \rho_{p}}{\partial t}+\nabla \cdot\left(\rho_{p} \mathbf{u}_{p}\right)=\nabla \cdot\left(k \nabla \rho_{p}\right)
$$

where $\mathbf{u}_{p}$ is the bulk particle velocity. The diffusion coefficient $k$ is assumed to be constant in this work. As a result, there is no mechanism to ensure that the particle concentration will not grow beyond close packing, which needs to be addressed in future works.

\subsection{Coupling}

Phase-coupling concerns the interaction between the phases. It includes the transfer of mass, momentum and energy between the phases [22]. For dilute particulate flows the interaction is often 
limited to the fluid exerting forces on the particles, referred to as one-way coupling. In dense flows one needs to consider that the hydrodynamic forces affect the fluid phase equally, which, when included, is referred to as back-coupling and subsequently leads to a model with two-way coupling. While there is no necessary exchange of mass between particles and fluid in a particulate flow, there is the displacement of fluid by the particles. In the VANSequations this is incorporated through the use of the porosity, which is entirely determined by the particle phase and given by

$\epsilon=1-\frac{\rho_{p}}{\tilde{\rho}_{p}}$.

$\tilde{\rho}_{p}$ is the material density of the particles, thus a particle's massvolume ratio, as opposed to the particle density $\rho_{p}$, which describes the presence of particulate mass within the two-phase flow.

The momentum exchange is given by the hydrodynamic force which may include, among others, drag force, pressure force, virtual-mass force. According to Newtons' second law, the force exerted by the fluid onto the particles is returned equally onto the fluid, that is

$\mathbf{F}_{f \rightarrow p}=-\mathbf{F}_{p \rightarrow f}$.

The choice $\mathbf{F}^{h}:=\mathbf{F}_{p \rightarrow f}$ leads to the signs in Eqs. (7) and (9).

\section{Discretization}

\subsection{The lattice Boltzmann method}

LBM can be understood to be a discretization approach to the Navier Stokes equations [23], the advection-diffusion equation or other target equations. It is centred around the probability distribution function $f$, that is defined on a discrete phase space, consisting of a regular grid $\Omega_{h}$ for spatial resolution and a limited number of accepted velocities $\xi_{i}$. Depending on the dimension $d$ and the number of allowed velocities $q$, the resulting scheme is denoted by $\mathrm{DdQ} q$. The expected mass in point $\mathbf{x}$ to move with velocity $\xi_{i}$ is then written as $f_{i}(\mathbf{x}, t)$ for $i \in\{0, \ldots, q-1\}$. by

The governing equation for the distribution function is given

$f_{i}\left(\mathbf{x}+\boldsymbol{\xi}_{i} \delta t, t+\delta t\right)-f_{i}(\mathbf{x}, t)=\frac{1}{\tau}\left(f_{i}^{e q}(\mathbf{x}, t)-f_{i}(\mathbf{x}, t)\right)$,

where $\delta t$ denotes the time step length. The right hand side of the equation is given by a BGK collision operator which includes the relaxation time $\tau$, related to the fluid's viscosity, as well as the equilibrium function $f_{i}^{e q}$, given by a discrete Maxwell-Boltzmann distribution

$f_{i}^{e q}=w_{i} \rho\left(1+\frac{\boldsymbol{\xi}_{i} \cdot \mathbf{u}}{c_{s}^{2}}+\frac{\left(\boldsymbol{\xi}_{i} \cdot \mathbf{u}\right)^{2}}{2 c_{s}^{4}}-\frac{\mathbf{u} \cdot \mathbf{u}}{2 c_{s}^{2}}\right)$,

with speed of sound $c_{s}$ and weights $w_{i}$.

The macroscopic quantities are recovered by taking the moments of $f_{i}$

$\sum_{i} f_{i}=\rho \quad \sum_{i} \xi f_{i}=\rho \mathbf{u}$,

while the pressure is represented through the density $p=\rho c_{s}^{2}$, making the LBM weakly compressible.

The standard LBM is implemented in a two-step scheme: Alternately, the left-hand side and the right-hand side are calculated in a streaming step

$f_{i}\left(\mathbf{x}+\xi_{i} \delta t, t+\delta t\right)=\hat{f}_{i}(\mathbf{x}, t)$

and a collision step

$\hat{f}_{i}(\mathbf{x}, t)=\left(1-\frac{1}{\tau}\right) f_{i}(\mathbf{x}, t)+\frac{1}{\tau} f_{i}^{e q}(\mathbf{x}, t)$.

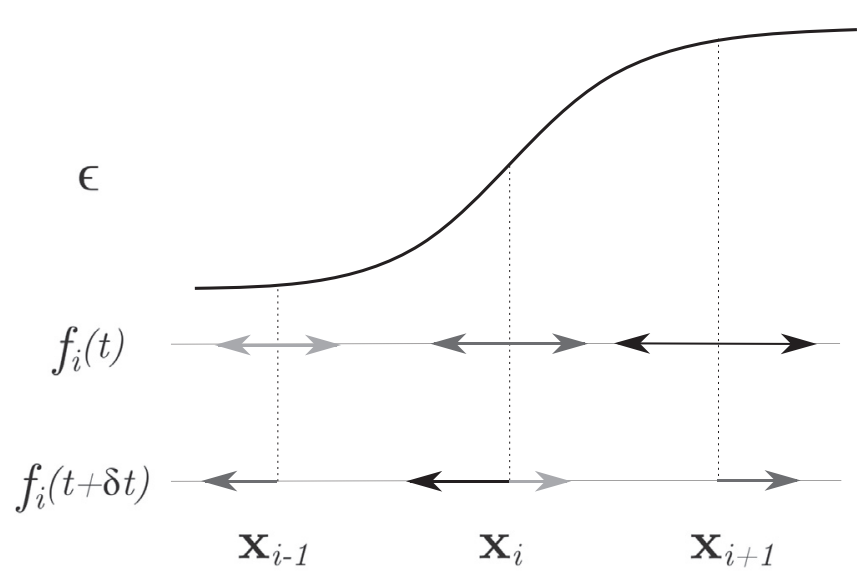

Fig. 1. Illustration of a streaming step for an LBM with scaled density. The top line represents the porosity, while the lower two lines show a distribution function before and after a streaming step. At time $t$, the fluid is at rest and the distributions functions are symmetric. After the streaming step at time $t+\delta t$ the central node has a distribution that is significantly slanted to the left, corresponding to an unwanted acceleration towards the region of low porosity.

The first incorporates the temporal shift of densities while the latter describes the redistribution of densities due to internal collisions in the fluid. Forces can furthermore be included through a scheme suggested by Guo et al. [20], by adapting the velocity in Eq. (6) and redistributing $f_{i}$ 's. In Section 4, a D2Q9 discretisation as well as a D3Q19 discretisation will be used. The corresponding values for $\boldsymbol{\xi}_{i}, w_{i}$ and $c_{s}$ can be found e.g. in [24].

\section{2. $L B M$ for the VANS equations}

To incorporate the similarity of the Navier-Stokes and VANS equations into an LBM scheme, Zhang et al. [18] suggest to scale the density function with the porosity. Accordingly, the density function is redefined to yield

$\sum_{i} f_{i}=\epsilon \rho \quad \sum_{i} \xi f_{i}=\epsilon \rho\langle\mathbf{u}\rangle$,

with the adapted equilibrium-function

$f_{i}^{e q}=\epsilon W_{i} \rho\left(1+\frac{\boldsymbol{\xi}_{i} \cdot\langle\mathbf{u}\rangle}{c_{s}^{2}}+\frac{\left(\boldsymbol{\xi}_{i} \cdot\langle\mathbf{u}\rangle\right)^{2}}{2 c_{s}^{4}}-\frac{\langle\mathbf{u}\rangle \cdot\langle\mathbf{u}\rangle}{2 c_{s}^{2}}\right)$.

Using Chapman-Enskog analysis, macroscopic equations can then be derived, yielding results similar to the VANS equations, but with an incorrect pressure term $\nabla(\epsilon p)$ [18]. In the case of an isobaric fluid at rest in a domain filled with a spatially fluctuating porosity, the pressure term $\nabla(\epsilon p)=p \nabla \epsilon$ would lead to a non-physical acceleration of the fluid. To remedy this, Zhang et al. suggest a correcting force term $\mathbf{F}^{p c}=p \nabla \epsilon$. But when investigated by Blais et al. [12] it was found not to adequately solve test cases with nonconstant porosity.

According to the authors of this paper, the issue with the approach lies in the usage of a correcting force term, which intervenes in the collision step. As illustrated in Fig. 1 for a simple onedimensional test case, the streaming step of an LBM with scaled density distributions skews the velocity. Therefore, the authors of this paper propose a modification of the streaming step to retrieve the correct pressure term (Fig. 2).

To achieve this, the density distribution is split into two parts which are streamed independently; one part given by $\pi_{i}=w_{i} \sum_{j} f_{j}$ and a remainder $v_{i}$, such that $f_{i}=\pi_{i}+v_{i}$. In rough terms, $\pi_{i}$ contains the density part of the distribution which is subsequently responsible for the pressure, whereas $v_{i}$ incorporates the remaining information. 


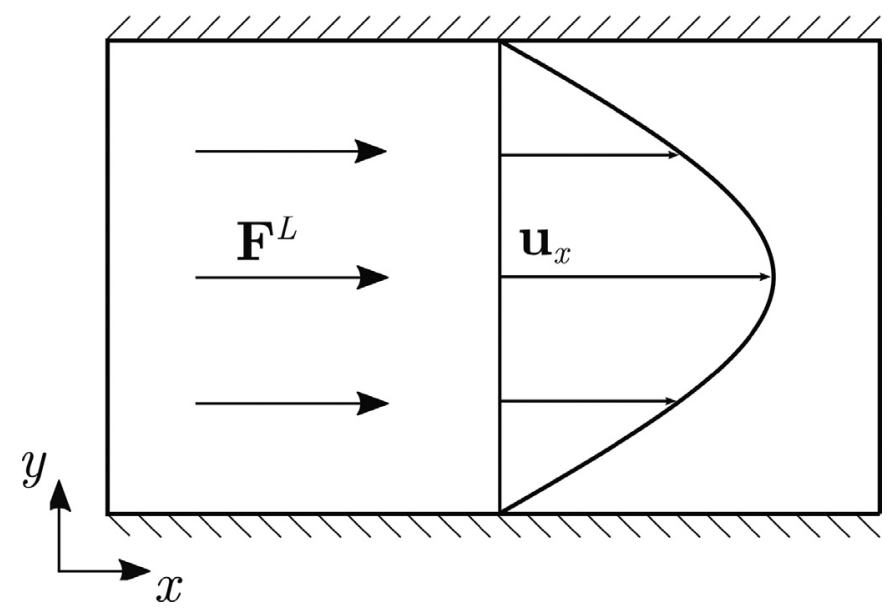

Fig. 2. Illustration of the Poiseuille test case. $\mathbf{F}^{L}$ is the driving force, while $\mathbf{u}_{x}$ schematically shows the profile of the velocity in $x$-direction and explicit forcing.

By scaling $\pi_{i}$ by $\frac{1}{\epsilon(\mathbf{x}, t)}$ before and rescaling it by $\epsilon(\mathbf{x}+\boldsymbol{\xi} \delta t, t+$ $\delta t)$ after the streaming step, the problem in the equilibrium case of Fig. 1 is adequately resolved. The rescaling procedure corresponds to an adaptation of the streaming step to

$$
\begin{aligned}
f_{i}\left(\mathbf{x}+\boldsymbol{\xi}_{i} \delta t, t+\delta t\right)= & \epsilon\left(\mathbf{x}+\boldsymbol{\xi}_{i} \delta t, t+\delta t\right) \frac{\pi_{i}(\mathbf{x}, t)}{\epsilon(\mathbf{x}, t)}+v_{i}(\mathbf{x}, t) \\
= & f_{i}(\mathbf{x}, t)+\pi_{i}(\mathbf{x}, t) \\
& \times \frac{\epsilon\left(\mathbf{x}+\boldsymbol{\xi}_{i} \delta t, t+\delta t\right)-\epsilon(\mathbf{x}, t)}{\epsilon(\mathbf{x}, t)},
\end{aligned}
$$

which, combined with the collision step, gives an adapted lattice Boltzmann equation

$$
\begin{aligned}
& f_{i}(\mathbf{x}+\boldsymbol{\xi} \delta t, t+\delta t)-f_{i}(\mathbf{x}, t) \\
& \quad=\frac{1}{\tau}\left(f_{i}^{e q}-f_{i}\right)+\pi_{i}(\mathbf{x}, t) \frac{\epsilon\left(\mathbf{x}+\xi_{i} \delta t, t+\delta t\right)-\epsilon(\mathbf{x}, t)}{\epsilon(\mathbf{x}, t)} .
\end{aligned}
$$

An investigation of this scheme in the hydrodynamic limit using Chapman-Enksog analysis is shown in Appendix A. Relying on the assumption that density fluctuations remain relatively small, the VANS momentum equations can be recovered.

\subsection{LBM scheme for the advection-diffusion equation}

The LBM with BGK collision operator can also be adapted to simulate the advection-diffusion equation. Using the density function $g_{i}$, the density can once again be recovered by $\rho_{p}=\sum_{i} g_{i}$. For the equilibrium function one can use $g_{i}^{e q}=w_{i} \rho_{p}\left(1+\frac{\xi_{i} \cdot \mathbf{u}_{p}}{c_{s}^{2}}\right)$, while the relaxation-time is chosen to be $\tau_{p}=k+\frac{\delta t}{2}$ and $\mathbf{u}_{p}$ is computed through a first order approximation

$$
\begin{aligned}
& \mathbf{u}_{p}(\mathbf{x}, t+\delta t) \\
& \quad=\mathbf{u}_{p}(\mathbf{x}, t)+\frac{1}{\rho_{p}}\left(-\nabla \cdot\left(\rho_{p} \mathbf{u}_{p}(\mathbf{x}, t) \mathbf{u}_{p}(\mathbf{x}, t)^{\top}\right)+(1-\epsilon) \mathbf{F}^{p}-\mathbf{F}^{h}\right),
\end{aligned}
$$

with body force $\mathbf{F}^{p}$. The authors of this paper refer to [25] for further details.

\subsection{Drag forces in $L B M$}

To include the force interaction between the fluid and particle phase, a forcing scheme by Guo et al. [20] is used. It requires a redistribution of the $f_{i}$ 's, as well as adapting the velocity used in the equilibrium distribution, as given by Eq. (7). This velocity $\mathbf{u}^{e q}$ is obtained by extrapolating the fluid's velocity to time $t+\frac{\delta t}{2}$ in accordance to Newton's second law

$\mathbf{u}^{e q}=\frac{1}{\epsilon \rho}\left(\sum_{i} \xi_{i} f_{i}+\frac{\delta t}{2}\left(\mathbf{F}^{h}+\epsilon \mathbf{F}^{f}\right)\right)$.

It should be noted that the hydrodynamic force generally includes the drag force, leading to an implicit relationship between the force-shifted velocity and velocity-dependent drag force. For simple drag laws this relationship can be resolved, which could improve accuracy as well as stability of some methods. Here, an approach by Guo and Zhao [17] for porous media with a quadratic drag law is extended to a dynamic particle phase. Therefore, the hydrodynamic force in this section is also assumed to be limited to a quadratic drag force

$\mathbf{F}^{h}=\mathbf{F}^{d}\left(\mathbf{u}_{r e l}\right)=-c_{0} \mathbf{u}_{\text {rel }}-c_{1} \mathbf{u}_{\text {rel }}\left|\mathbf{u}_{\text {rel }}\right|$,

with parameters $c_{0}$ and $c_{1}$ and $\mathbf{u}_{r e l}=\mathbf{u}-\mathbf{u}_{p}$. For drag laws which are not of this form, a second order approximation could be used in order to implement the scheme.

By introducing the extrapolated particle velocity $\mathbf{u}_{p}^{e q}:=\mathbf{u}_{p}+$ $\frac{\delta t}{2 \rho_{p}}\left(-\nabla \cdot\left(\rho_{p} \mathbf{u}_{p} \mathbf{u}_{p}^{\top}\right)+(1-\epsilon) \mathbf{F}^{p}-\mathbf{F}^{d}\left(\mathbf{u}_{r e l}^{e q}\right)\right)$ and extrapolated relative velocity $\mathbf{u}_{r e l}^{e q}:=\mathbf{u}^{e q}-\mathbf{u}_{p}^{e q}$, Eq. (10) can be adapted to yield

$$
\begin{aligned}
\mathbf{u}^{e q} & =\frac{1}{\epsilon \rho}\left(\sum_{i} \xi_{i} f_{i}+\frac{\delta t}{2} \epsilon \mathbf{F}^{f}+\frac{\delta t}{2} \mathbf{F}^{d}\left(\mathbf{u}_{r e l}^{e q}\right)\right) \\
& =\frac{1}{\epsilon \rho}\left(\sum_{i} \xi_{i} f_{i}+\frac{\delta t}{2} \epsilon \mathbf{F}^{f}+\frac{\delta t}{2}\left(-c_{0} \mathbf{u}_{r e l}^{e q}-c_{1} \mathbf{u}_{r e l}^{e q}\left|\mathbf{u}_{r e l}^{e q}\right|\right)\right) .
\end{aligned}
$$

Subtracting the extrapolated particle velocity $\mathbf{u}_{p}^{e q}$ from both sides, gives an implicit equation for $\mathbf{u}_{r e l}^{e q}$

$$
\begin{aligned}
\mathbf{u}_{r e l}^{e q}= & \frac{1}{\epsilon \rho} \sum_{i} \boldsymbol{\xi}_{i} f_{i}+\frac{\delta t}{2 \rho} \mathbf{F}^{f}-\mathbf{u}_{p}+\frac{\delta t}{2} \nabla \cdot\left(\rho_{p} \mathbf{u}_{p} \mathbf{u}_{p}^{\top}\right) \\
& -\frac{\delta t}{2 \tilde{\rho}_{p}} \mathbf{F}^{p}+\frac{\delta t}{2}\left(\frac{1}{\epsilon \rho}-\frac{1}{\rho_{p}}\right)\left(-c_{0} \mathbf{u}_{r e l}^{e q}-c_{1} \mathbf{u}_{r e l}^{e q}\left|\mathbf{u}_{r e l}\right|\right) .
\end{aligned}
$$

Through the introduction of an auxiliary variable

$\mathbf{v}:=\frac{1}{\epsilon \rho} \sum_{i} \boldsymbol{\xi}_{i} f_{i}+\frac{\delta t}{2 \rho} \mathbf{F}^{f}-\mathbf{u}_{p}+\frac{\delta t}{2 \rho_{p}} \nabla \cdot\left(\rho_{p} \mathbf{u}_{p} \mathbf{u}_{p}^{\top}\right)-\frac{\delta t}{2 \tilde{\rho}_{p}} \mathbf{F}^{p}$

which includes all velocity-independent terms, this relation can be explicitly solved [17] by

$\mathbf{u}_{r e l}^{e q}=\frac{\mathbf{v}}{c_{0}^{*}+\sqrt{c_{0}^{* 2}+c_{1}^{*}|\mathbf{v}|}}$

where $c_{0}^{*}=\frac{1}{2}\left(1+\frac{\delta t}{2}\left(\frac{1}{\rho}-\frac{1}{\rho_{p}}\right) c_{0}\right)$ and $c_{1}^{*}=\frac{\delta t}{2}\left(\frac{1}{\rho}-\frac{1}{\rho_{p}}\right) c_{1}$. The drag force can accordingly be reconstructed by

$\mathbf{F}^{d}\left(\mathbf{u}_{r e l}^{e q}\right)=-c_{0} \mathbf{u}_{r e l}^{e q}-c_{1} \mathbf{u}_{r e l}^{e q}\left|\mathbf{u}_{r e l}^{e q}\right|$.

\subsection{Euler-Euler VANS LBM}

By combining the VANS LBM from Section 3.2 and the advection-diffusion LBM from Section 3.3 the Euler-Euler VANS LBM is formed. For each time step, the following steps are executed:

(i) $\epsilon$ is determined from $\rho_{p}$ according to Eq. (4)

(ii) the hydrodynamic and body forces are determined

(iii) the new equilibrium velocity is determined (Eq. (12) or Eq. (13))

(iv) the particle velocity is adapted according to (9)

(v) fluid and particles perform collide and stream 
Steps (ii) and (iii) are implemented for two different schemes. One takes the implicit drag-velocity relation from Section 3.4 into consideration while the other does not. The first variant, in which the velocity is simply shifted according to Eq. (10) will be referred to as explicit forcing scheme. The drag force in (ii) will be determined at time $t$, using $\mathbf{u}_{r e l}=\mathbf{u}-\mathbf{u}_{p}$. Accordingly, the equilibrium velocity in step (iii) will be given by

$\mathbf{u}^{e q}=\frac{1}{\epsilon \rho}\left(\sum_{i} \xi_{i} f_{i}+\frac{\delta t}{2} \mathbf{F}^{h}\left(\mathbf{u}-\mathbf{u}_{p}\right)+\frac{\delta t}{2} \epsilon \mathbf{F}^{f}\right)$.

For the implicit forcing scheme, the drag force in (ii) is determined using $\mathbf{u}_{r e l}^{e q}$ from Eq. (11). The velocity for the equilibrium distribution in step (iii) is then recovered using the definition of $\mathbf{u}_{\text {rel }}^{e q}$, that is

$$
\begin{aligned}
\mathbf{u}^{e q} & =\mathbf{u}_{r e l}^{e q}+\mathbf{u}_{p}^{e q} \\
& =\mathbf{u}_{r e l}^{e q}+\mathbf{u}_{p}+\frac{\delta t}{2 \rho_{p}}\left(-\nabla \cdot\left(\rho_{p} \mathbf{u}_{p} \mathbf{u}_{p}^{\top}\right)+(1-\epsilon) \mathbf{F}^{p}-\mathbf{F}^{d}\left(\mathbf{u}_{r e l}^{e q}\right)\right) .
\end{aligned}
$$

\section{Results}

To validate the novel VANS LBM scheme, four simple twodimensional test cases with analytical solutions are examined. The first two are fluid flows through a homogeneous, static particle phase and follow the example of Guo and Zhang [17]. The third case is a periodic fluid flow through a square box filled with a static fluid phase, but with a porosity that fluctuates sinusoidally in direction of the flow, which is relevant for correctly validating the pressure term. The fourth test case investigates the fluid flow through a moving particle phase with a sinusoidal concentration.

In the last test case, the Euler-Euler VANS LBM is assessed by simulating a Rayleigh-Taylor instability. All two-dimensional test cases rely on a D2Q9 discretisation, whereas the Rayleigh-Taylor test case uses a D3Q19 discretisation for the fluid phase and a D3Q7 discretisation for the particle phase. All test cases are implemented for both implicit and explicit forcing.

\subsection{Porous Poiseuille flow}

The plane Poiseuille flow is a flow bounded by two infinitely extending planes. Near the boundary planes, the fluid is slowed down due to the application of no-slip boundaries, leading to a parabolic flow pattern for one-phase flows. In the generalized version of the Poiseuille flow investigated in this section, drag forces are added and the influence of the porosity is included, leading to a slightly different steady-state solution, which will be stated subsequently.

The simulation of this test case is carried out in a nondimensional domain of size $2 \times 1$. Boundary conditions in $x$ direction are chosen to be periodic, while a bounce-back boundary condition is imposed at height $y=0$ and $y=1$. The Reynolds number $\operatorname{Re}$ is chosen to be 10 and the channel is filled with a constant particle presence, yielding a porosity $\epsilon=0.9$. A constant force $\mathbf{F}^{f}$ in $x$-direction drives the flow, while a linear drag force $\mathbf{F}^{d}=-(1-\epsilon) c_{0}\langle\mathbf{u}\rangle$ with $c_{0}=10$ counteracts it, resulting in a symmetric steady-state velocity, which can analytically be determined [17] to be

$\mathbf{u}^{s}(y)=\mathbf{u}_{r}\left(1-\frac{\cosh (r(y-1 / 2))}{\cosh (r / 2)}\right)$,

with $r=\sqrt{\operatorname{Re} c_{0}}$ and reference velocity $\mathbf{u}_{r}=\frac{\epsilon}{(1-\epsilon) c_{0}} \mathbf{F}^{f}$. In order to normalize the reference velocity to one, $\mathbf{F}^{f}$ is chosen to be $\left(\frac{(1-\epsilon) c_{0}}{\epsilon}, 0\right)$. The simulation runs until $t=20$, when a steady-state has been achieved, for discretisation lengths $\delta x$ ranging from $\frac{1}{10}$ to

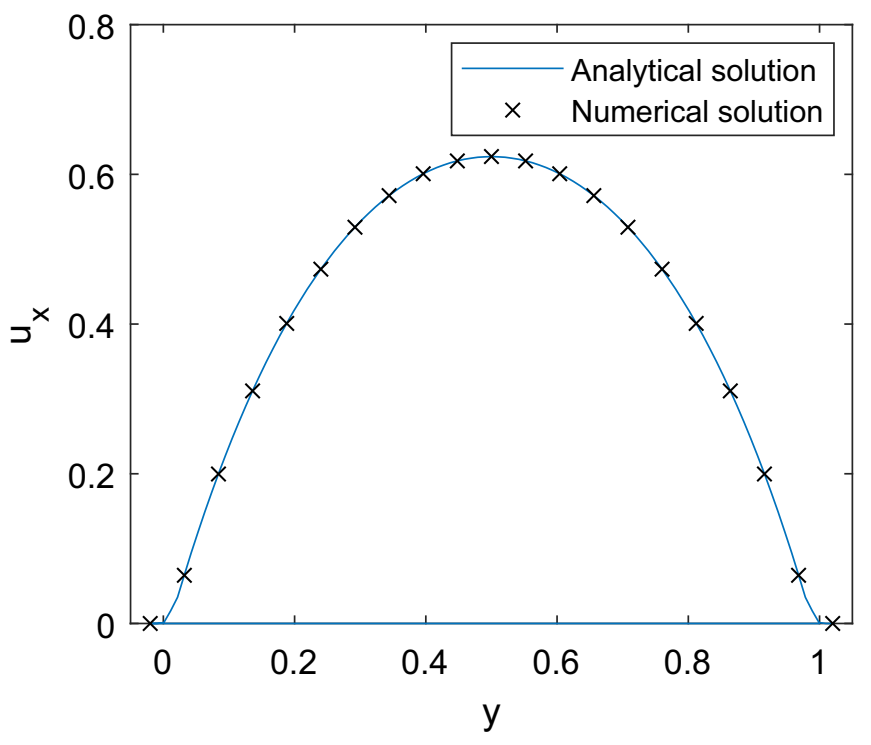

Fig. 3. Velocity profile of the Poiseuille test case for mesh resolution $\delta x=\frac{1}{50}$.

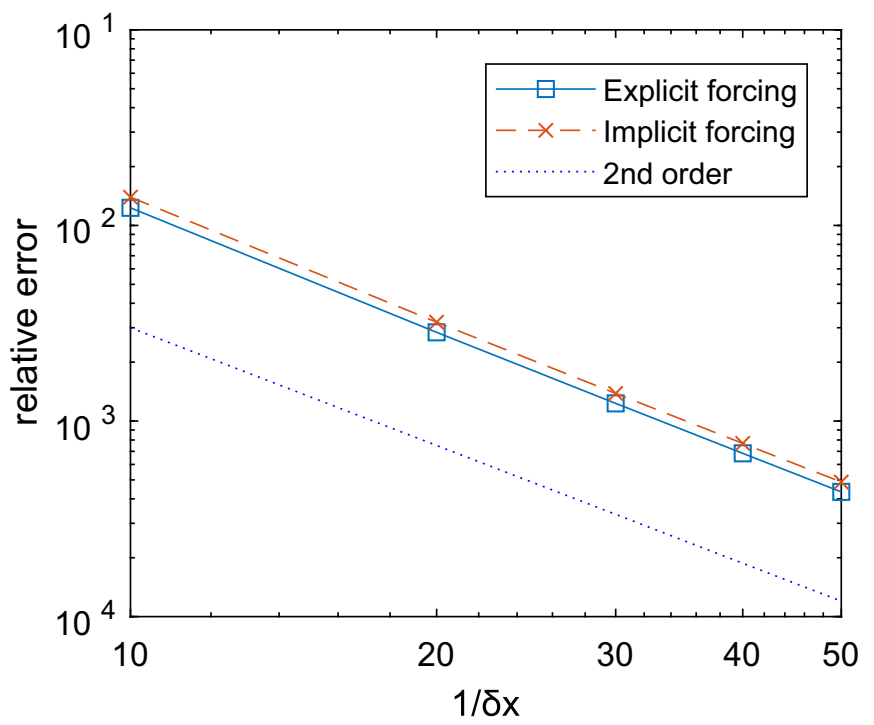

Fig. 4. Relative error of the Poiseuille test case for different mesh resolutions $\delta x$.

$\frac{1}{50}$. In Fig. 3 the profile of the $x$-component of the velocity of both the analytical and numerical solution is plotted over the height of the channel. As shown, the velocity profile for the Poiseuille test case is in excellent agreement with the analytical solution. In Fig. 4 one finds the relative error plotted over the inverse discretisation length. It can be seen that both the explicit and implicit forcing scheme from Section 3.5 yield a second order convergence, while the explicit forcing scheme performs marginally better.

In fact, the second order convergence is expected seeing that for $\epsilon=$ const one gets $\nabla(\epsilon p)=\epsilon \nabla p$. For the same reason, the rescaling procedure in the streaming step yields the same results as the conventional streaming procedure and accordingly the convergence should match that of the standard LBM.

\subsection{Porous couette flow}

The Couette-flow is a flow bounded by two planes which move relative to each other. For one-phase flows this leads to a linear velocity profile between the planes. Through the inclusion of drag forces and porosity, the generalised Couette flow in this section 


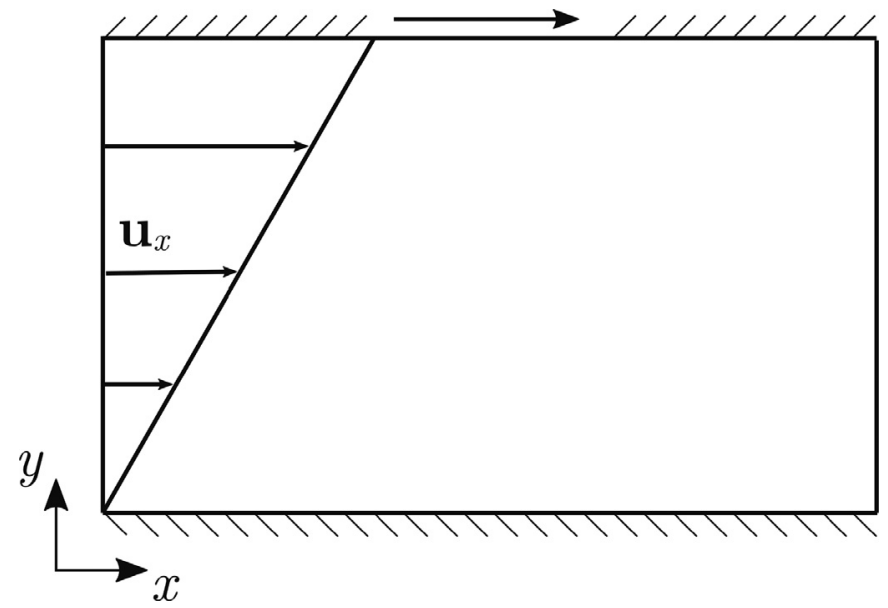

Fig. 5. Illustration of the Couette test case. The upper plane deives the flow and $\mathbf{u}_{x}$ schematically shows the velocity in $x$-direction.

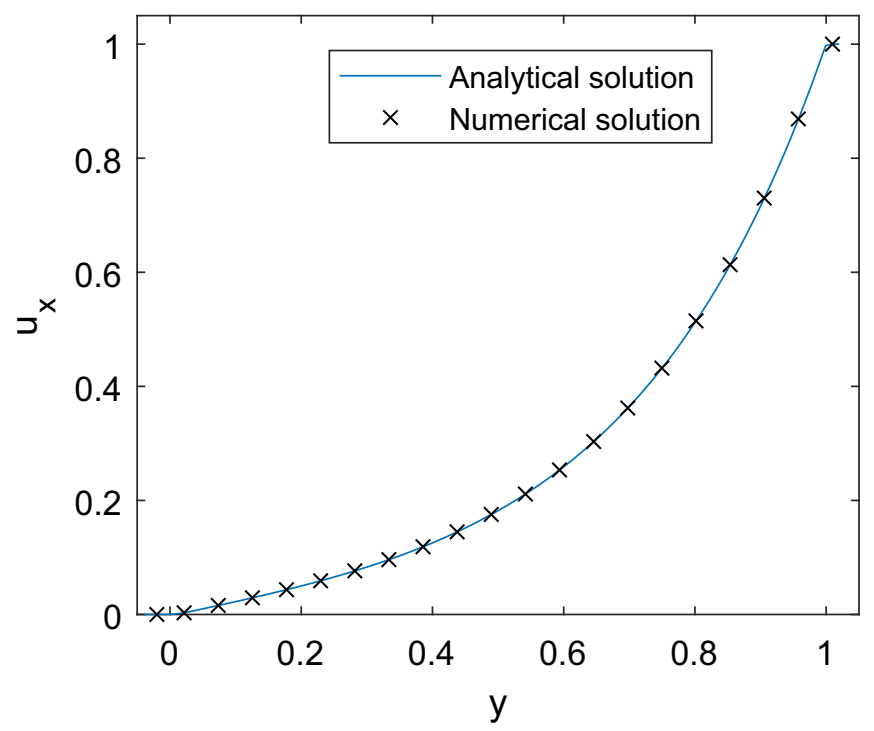

Fig. 6. Velocity profile of the Couette test case for mesh resolution $\delta x=\frac{1}{50}$ and explicit forcing.

once more has a slightly different solution. Just as in the first test case, the $2 \times 1$ channel has a constant porosity $\epsilon=0.9$ and a linear drag law with $c_{0}=10$. Unlike the first test case, the flow is not driven by a force, but by the moving upper boundary plane as illustrated in Fig. 5. On the lower boundary plane, at $y=0$, a bounce-back condition is imposed, the upper plane at $y=1$ has a velocity boundary with velocity $\mathbf{v}=(1,0)$. In $x$-direction periodic boundary conditions are imposed. The steady-state solution for this case is then given by Guo and Zhao [17]

$\mathbf{u}^{s}(y)=\mathbf{v} \frac{\sinh (r y)}{\sinh (r)}$,

with $r=\sqrt{\operatorname{Re} \frac{1-\epsilon}{\epsilon} c_{0}}$.

In Fig. 6 we find the velocity-component in $x$-direction of both the numerical and analytical solution plotted over the height of the channel. Again, the two solutions are in excellent agreement. In Fig. 7 the relative error is plotted over the inverse discretisation length. Considering that the test case again uses a constant porosity, the same reasoning as for the Poiseuille test case holds true and once more a second order convergence for both forcing schemes can be observed.

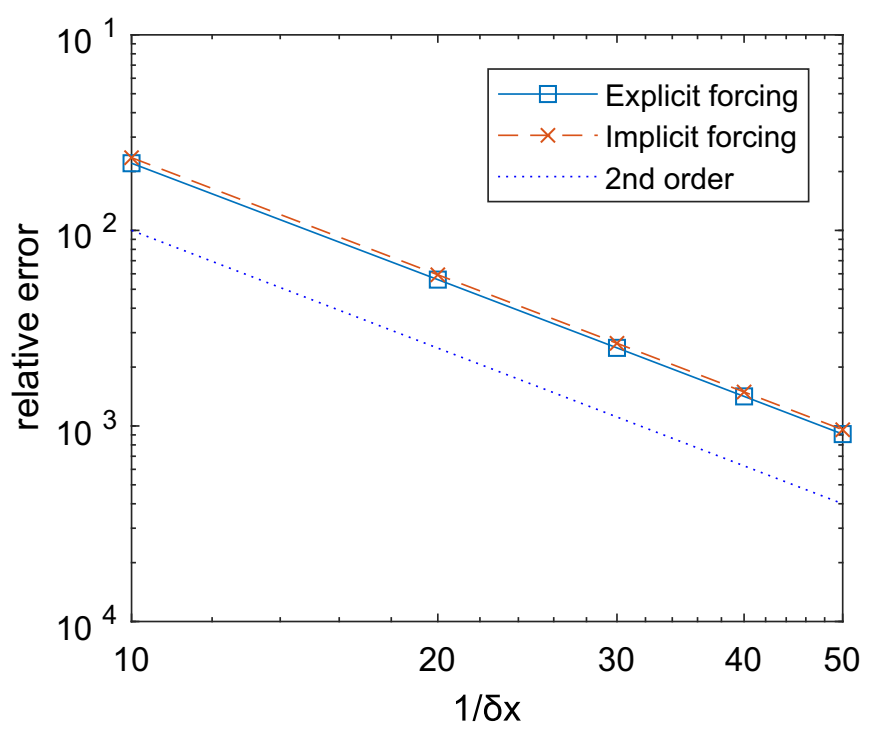

Fig. 7. Relative error of the Couette test case for different mesh resolutions $\delta x$.

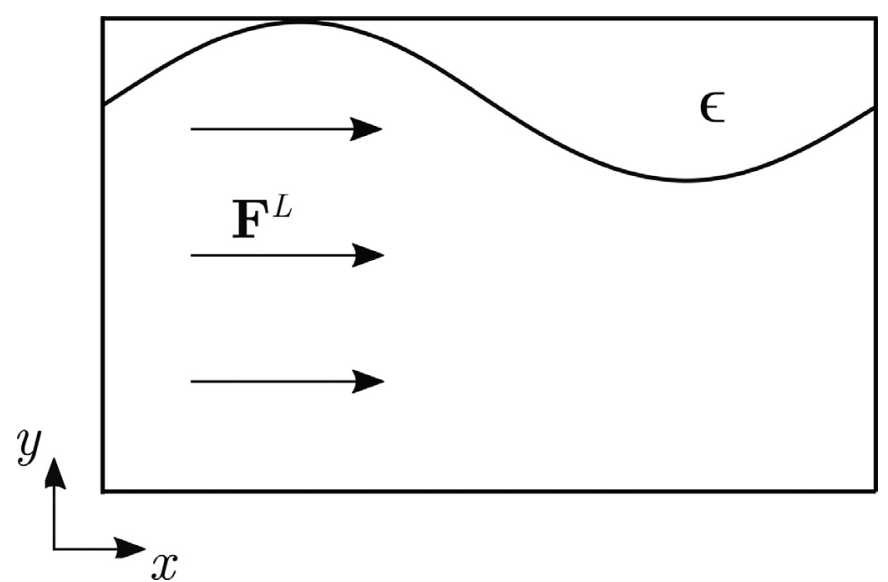

Fig. 8. Illustration of the variable porosity test case. The upper curve represents the spatial fluctuation of $\epsilon$ over the length of the channel. A Force $\mathbf{F}^{L}$ drives the flow.

\subsection{Variable porosity in space}

This test case was chosen in order to determine the performance of the scheme in the presence of porosity gradients and consequently to validate the pressure term. The domain is given by a square channel with dimensions $2 \times 1$. The porosity varies sinusoidally over the length of the channel and is chosen to be $\epsilon(x)=1+a(\sin (\pi x)-1)$, with $a=0.1$. The flow is driven by a constant force $\mathbf{F}^{f}$ in $x$-direction and the boundaries are chosen to be periodic, thus the driving force is solely counteracted by the drag force given by $\mathbf{F}^{d}=\epsilon^{2}(1-\epsilon)\langle\mathbf{u}\rangle$. While this drag would not correspond to any physical drag law (due to the cubic influence of the porosity), it does allow for the derivation of a simple analytical steady-state solution, namely $\mathbf{u}^{S}=\frac{\mathbf{F}^{f}}{\epsilon a c_{0}}$. Accordingly, the driving force is chosen to be $\mathbf{F}^{f}=a c_{0}$ to obtain the solution $\mathbf{u}^{S}=\frac{1}{\epsilon}$.

A schematic representation of the test case has been included in Fig. 8, where the curve of the porosity has been superimposed over the channel.

In Fig. 9 the velocity in $x$-direction is plotted over the length of the channel. We once more find excellent agreement with the analytical solution. For both forcing schemes there is a second order convergence as can be observed in Fig. 10, where the relative error is plotted over the inverse discretisation length. 


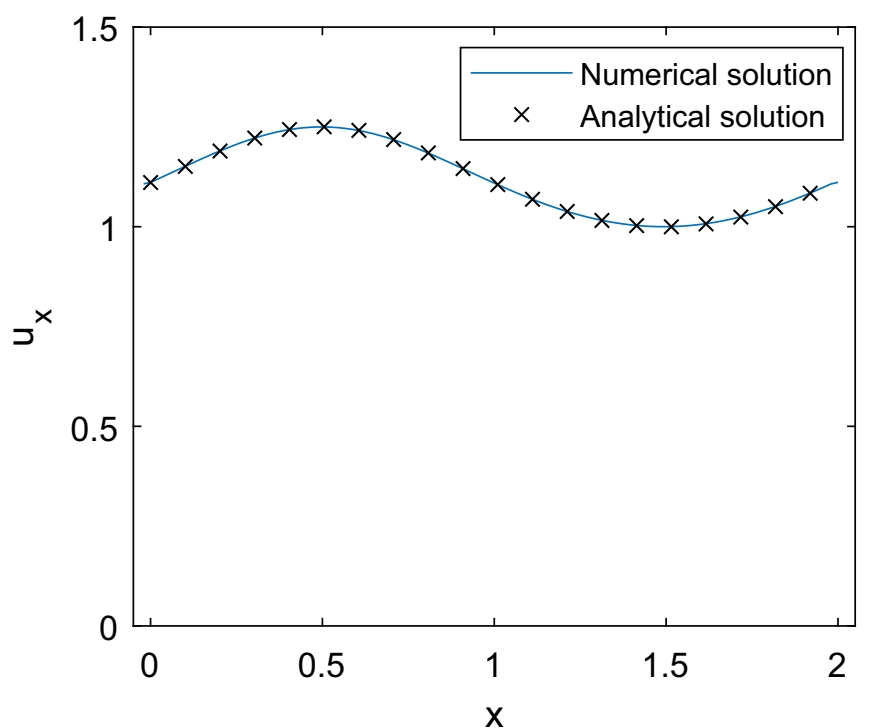

Fig. 9. Velocity profile of the variable porosity test case for mesh resolution $\delta x=\frac{1}{50}$ and explicit forcing.

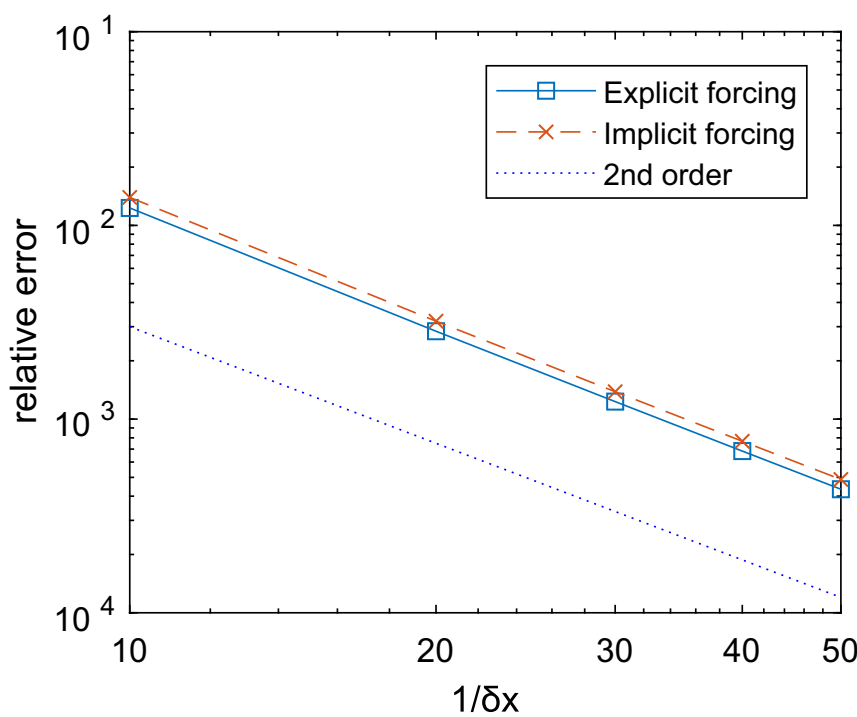

Fig. 10. Relative error of the variable porosity test case for different mesh resolution $\delta x$.

\subsection{Variable porosity in space and time}

In order to investigate the performance for temporally fluctuating porosities, the previous test case was also implemented for a particle phase moving with a constant velocity $\mathbf{u}_{p}=(0.5,0)$. As a result, the porosity is given by $\epsilon(x, t)=1+a(\sin (\pi(x-0.5 t))-$ 1) and the drag law is given by $\mathbf{F}^{d}=\epsilon^{2}(1-\epsilon) c_{0}\left(\langle\mathbf{u}\rangle-\mathbf{u}_{p}\right) . a, c_{0}$ and $\mathbf{F}^{f}$, as well as the boundary conditions remain unchanged. The corresponding analytical solution is given by $\mathbf{u}^{S}=\mathbf{u}_{p}+\frac{1}{\epsilon}$.

The test case is illustrated in Fig. 11, where the porosity at two points in time has been superimposed in order to illustrate the temporal development of the porosity. In Fig. 12, the $x$-component of the analytical and simulated velocity is plotted over the length of the channel for time $t=20$. As shown, the numerical results are in good agreement with the analytical solution. From Fig. 13 it can be seen that the relative error is of the order of magnitude $10^{-3}$ which, while small, seems to be a systematic error.

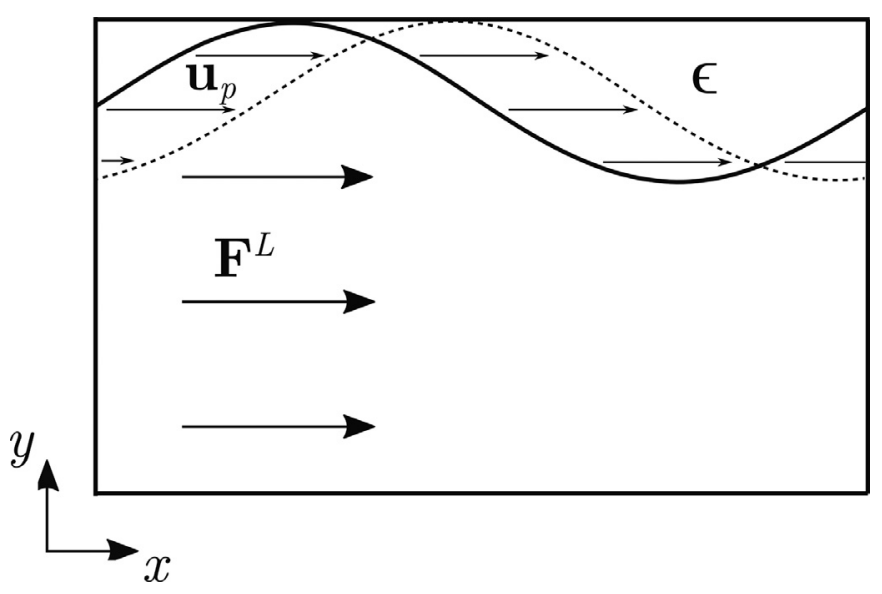

Fig. 11. Illustration of the moving variable porosity test case. The solid curve represents the spatial fluctuation of $\epsilon$ over the length of the channel, as does the dotted line for some later time. A Force $\mathbf{F}^{L}$ drives the flow.

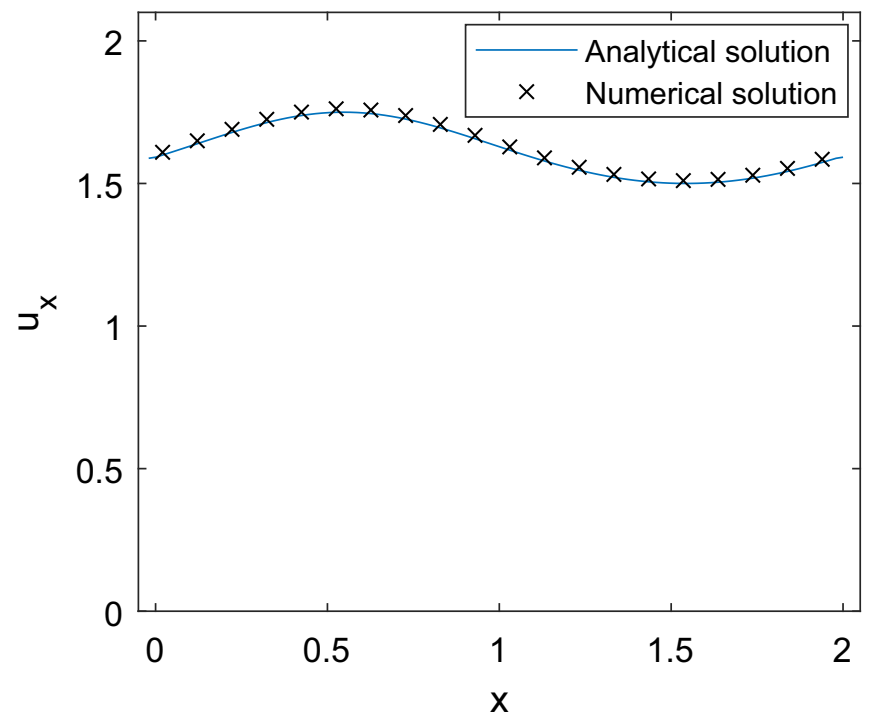

Fig. 12. Velocity profile of moving variable porosity test case for mesh resolution $\delta x=\frac{1}{50}$ and explicit forcing.

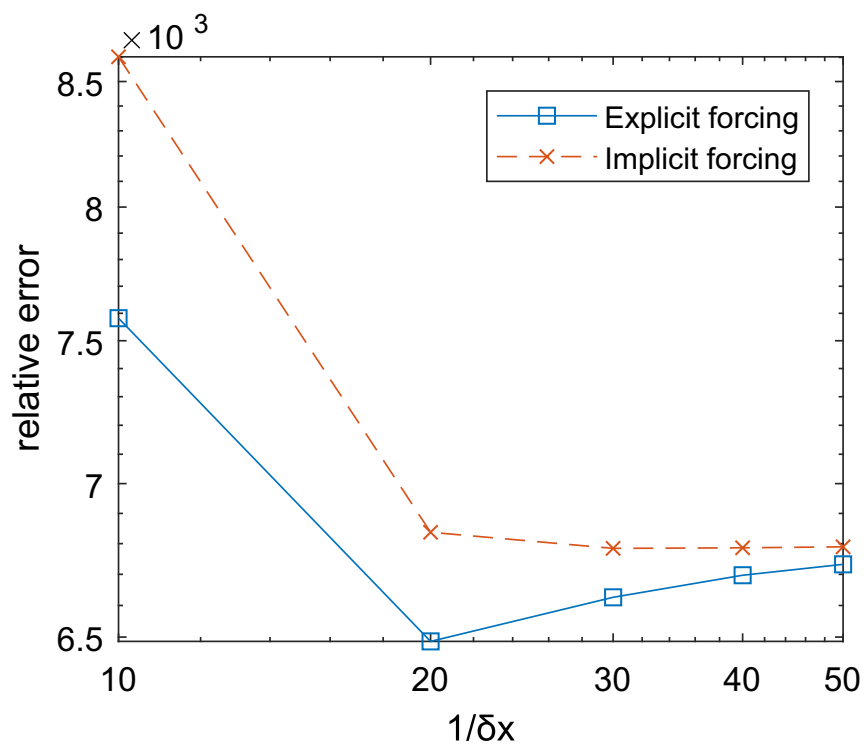

Fig. 13. Relative error of the moving variable porosity test case for different mesh resolutions $\delta x$. 


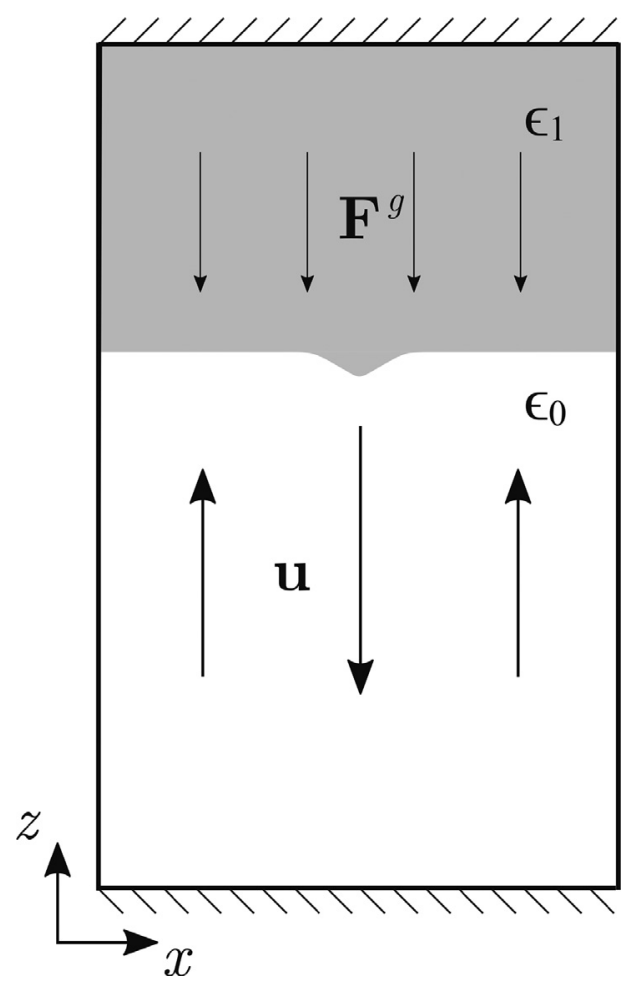

Fig. 14. Illustration of the initial setting of the Rayleigh-Taylor test case at the $y=0.00075 \mathrm{~m}$ plane. Dark regions $\left(\epsilon_{1}\right)$ represent regions of high particle concentration, while light regions $\left(\epsilon_{0}\right)$ represent pure fluid. The effective gravitational force $\mathbf{F}_{g}$ accelerates particles downwards, growing the perturbation at the center. $\mathbf{u}$ represents the general flow direction of the test case: The perturbation moves downwards through the center, while pure fluid moves upward at the sides of the domain.

\subsection{Rayleigh-Taylor}

A Rayleigh-Taylor instability occurs when a heavy fluid is placed over a lighter fluid. Gravitational forces accelerate the heavy fluid downward. In small perturbations, this leads to an acceler- ation of the surrounding fluid towards the perturbation, consequently leading to its growth. As a result, the bulk of the upper mass moves through these perturbations, while the light fluid moves upwards around it, leading to high shear rates and vortices at the fluids' interface, similar to a Kelvin-Helmholtz instability.

The phenomenon can also be observed when one substitutes the heavier fluid phase with a suspension consisting of a light fluid and heavy particles. This suspension behaves similar to a fluid, leading to the same phenomenon.

For this test case this was realized in a threedimensional simulation of a channel with dimensions $0.0015 \mathrm{~m} \times 0.0015 \mathrm{~m} \times 0.0045 \mathrm{~m}$. At $z=0 \mathrm{~m}$ and $z=0.0045 \mathrm{~m}$ a bounce-back boundary condition is imposed, while in $x$ and $y$-direction periodic boundary conditions are chosen. The fluid phase has a kinematic viscosity $\mu_{f}=1 \cdot 10^{-4} \frac{\mathrm{kg}}{\mathrm{ms}}$ and density $\rho=1000 \frac{\mathrm{kg}}{\mathrm{m}^{3}}$.

The top fifth of the channel is occupied by 10.000 .000 spherical particles of radius $r=2 \cdot 10^{-6} \mathrm{~m}$ and density $\rho_{p}=1010 \frac{\mathrm{kg}}{\mathrm{m}^{3}}$, leading to an initial porosity of $\epsilon_{1} \approx 0.85$. Additionally, a small spherical perturbation of particle-rich fluid is introduced, centred around $(0.00075 \mathrm{~m}, 0.00075 \mathrm{~m}, 0.0036 \mathrm{~m})$ with radius $4.5 \cdot 10^{-5} \mathrm{~m}$.

The particles' motion is simulated using the advection-diffusion model, as described in Chapter 3.3 using the diffusion coefficient $k=8 \cdot 10^{-9} \frac{\mathrm{m}^{2}}{\mathrm{~s}}$.

The flow is driven by the gravity force on the particles, counteracted by the buoyancy force, leading to an effective force $\mathbf{F}^{p}=$ $(1-\epsilon)\left(\rho-\tilde{\rho}_{p}\right) \mathbf{g}$, where $\mathbf{g}=(0,0,-9.81) \frac{\mathrm{m}}{\mathrm{s}^{2}}$ is the gravitational acceleration.

The phase interaction is restricted to the Stokes' drag

$\mathbf{F}^{d}=(1-\epsilon) \frac{9 \rho v_{f}}{2 r^{2}}\left(\langle\mathbf{u}\rangle-\mathbf{u}_{p}\right)$.

The initial setup of this test case is schematically depicted in Fig. 14. The discretisation lengths are given by $\delta x=2.5 \cdot 10^{-5} \mathrm{~m}$ and $\delta t=1.459 \cdot 10^{-4} \mathrm{~s}$. The Péclet-number can be determined to be $\mathrm{Pe}=\frac{0.0045 \mathrm{~m}}{k} \sqrt{|\mathbf{g}| 0.0045 \mathrm{~m}\left(\rho-\tilde{\rho}_{p}\right) / \tilde{\rho}_{p}} \approx 53$.

For the explicit forcing scheme no stable results were obtained. As depicted in Fig. 15, the particle concentration contains large oscillations, which grow and cause extreme density fluctuations within a few time steps. The implicit forcing scheme however

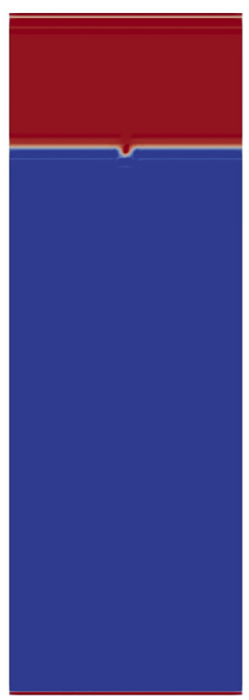

(a) $\mathrm{t}=4 \delta t$

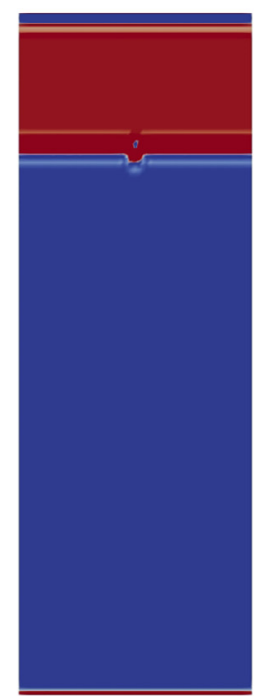

(b) $\mathrm{t}=5 \delta t$
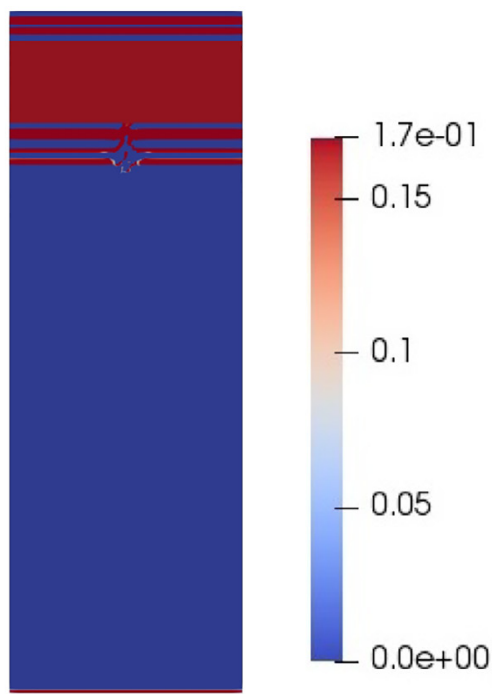

(c) $\mathrm{t}=6 \delta t$

Fig. 15. Particle density $\rho_{p}$ scaled by a factor $\frac{1}{1000}$ for the explicit forcing scheme illustrated on the plane $x=0.00075 \mathrm{~m}$ for different time steps. $t=5 \delta t t=6 \delta t$ include the oscillations, that cause the scheme to be unstable. 


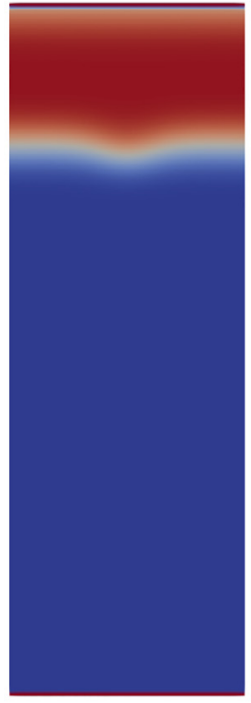

(a) $t=1 \mathrm{~s}$

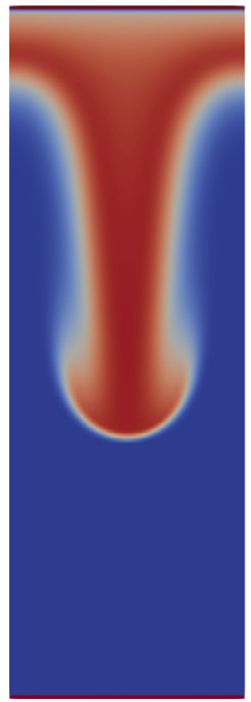

(d) $t=2.5 \mathrm{~s}$

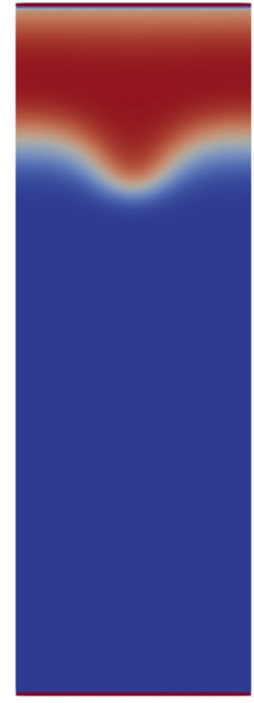

(b) $t=1.5 \mathrm{~s}$

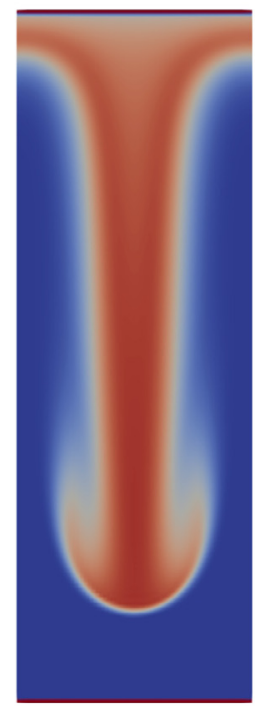

(e) $t=3 \mathrm{~s}$
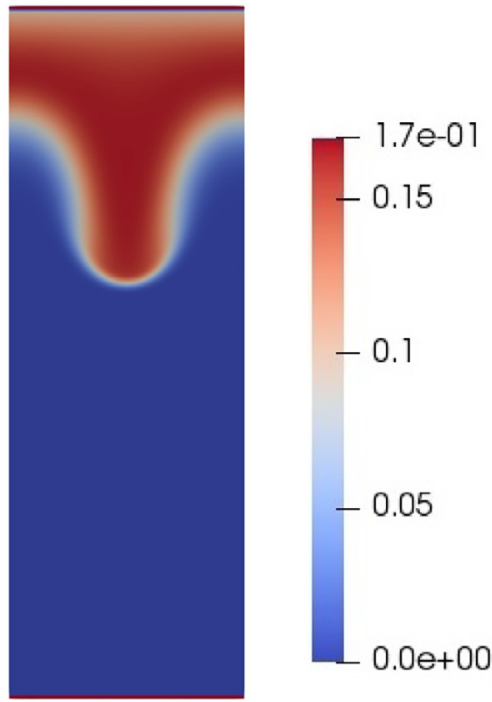

(c) $t=2 \mathrm{~s}$

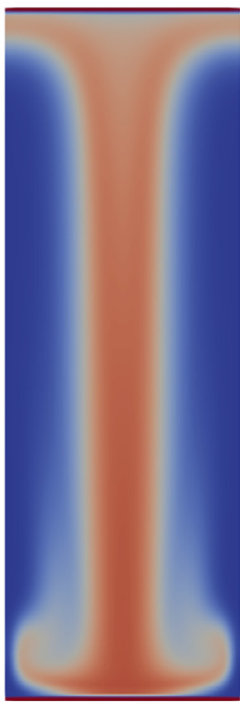

(f) $t=3.5 \mathrm{~s}$

Fig. 16. Particle density $\rho_{p}$ scaled by a factor $\frac{1}{1000}$ for the implicit forcing scheme illustrated on the plane $x=0.00075 \mathrm{~m}$ for different time steps. From time $1 \mathrm{~s}$ to $2 \mathrm{~s}$, the growth of the protrusion can be observed. $t=2.5 \mathrm{~s}$ to $3.5 \mathrm{~s}$ show the unfolding of the protrusion, as well as the circulatory motion accelerating the outer particles upwards.

proves to be more stable. Fig. 16 shows the temporal development of the particle concentration. Blue color indicates regions of low particle concentrations or high porosity (e.g. $\epsilon \approx 1$ ), whereas red indicates regions of high particle concentration or low porosity (e.g. $\epsilon \approx 0.83$ ).

As depicted in Fig. 16(a), the particle concentrations still closely resembles the initial setting with the perturbation at the centre, although diffusion blurred the boundary between clear and particleladen fluid. At time $t=1.5 \mathrm{~s}$ (Fig. 16(b)) the perturbation slowly grows, while the particle concentration next to it starts to decrease. In Fig. 16(c) at time $t=2 \mathrm{~s}$, one can observe an increased rate of growth of the protrusion which almost contains the bulk of particle mass. In Fig. 16(d) of a secondary instability starts to form at the tip of the protrusion, similar to a Kelvin-Helmholtz instability. It is formed by the downward motion of the particle-laden fluid and upward motion of the pure fluid around it, causing high shear stresses. In Fig. 16(e) nearly all particles move downwards through the centre, while the instability at the tip of the protru- sion continues growing. At time $t=3.5 \mathrm{~s}$ (Fig. 16(e)) a portion of the particle load has reached the bottom, while the outer parts of the protrusion are accelerated outward and upward due to the circulatory motion of the fluid in the domain.

The qualitative motion of the particle concentration is in agreement with Zhang's simulation of a sedimentation process using Lagrangian particles [26] as one can clearly discern the acceleration of particles through the middle, the unfolding of the head of the protrusion, as well as the recirculation upwards after the motion.

\section{Conclusion}

In this paper, a Euler-Euler LBM for particulate flows is introduced which incorporates the displacement of fluid by particles. For the fluid phase the volume-averaged Navier-Stokes equations are used, in which the presence of particles is incorporated through the use of the fluid's volume fraction $\epsilon$, commonly referred to as porosity. For the particles an advection-diffusion model 
with constant diffusion coefficient is chosen. The coupling approach between the phases consists of the porosity's dependency on $\rho_{p}$ and drag forces acting on both particle and fluid according to Newton's second law. To improve stability, an adapted forcing scheme for quadratic drag laws is introduced in Section 3.4. In order to obtain an LBM for the VANS equations, an adapted streaming step is proposed, which scales part of the probability density function $f_{i}$ for the fluid phase, depending on the local porosity.

This novel VANS LBM's behavior in the hydrodynamic limit is investigated in Appendix A. While the mass conservation equation is slightly different to that of the VANS equations, the scaling procedure in the streaming step ensures the correct density for incompressible flows. The momentum conservation equation relies on the assumption that fluctuations in pressure remain small whenever there are large porosity gradients. With this assumption, the momentum equation of the VANS equations is in fact retrieved in the hydrodynamic limit.

The performance of the novel VANS LBM and the Euler-Euler VANS LBM for particulate flows is tested in Section 4. In four simple test cases with analytical solutions, it is shown that the VANS LBM has second order convergence in the presence of a static particle-phase for both the explicit forcing scheme (explicit) as well as the implicit forcing scheme, even in the presence of porosity gradients. For the non-static test case one finds that the results are in good agreement with the analytical solution, however, no convergence is obtained. The analytical solution can be recovered up to an error of $6 \cdot 10^{-3}$. While the explicit forcing scheme yields better results than the implicit scheme in the analytical test cases, it proves to be unstable for the Euler-Euler model as shown in the Rayleigh-Taylor test case. For the implicit forcing scheme, the temporal development of the concentration closely resembles other simulations of Rayleigh-Taylor instabilities. Since the test case heavily depends on fluid-particle interaction, it can be assumed, that Euler-Euler VANS LBM accurately incorporates the coupling. To fully capture the physics of dense particulate flows, some additional modifications of the Euler-Euler VANS LBM scheme are required. Other hydrodynamic interactions such as shear induced forces, virtual mass forces and other drag laws could easily be included. The subgrid-stress could be incorporated using a Smagorinsky model. Extending the VANS scheme to compressible fluid would also greatly improve its usability. The biggest hurdle towards an implementation for dense particulate flow lies in the behaviour near close-packing of the advection-diffusion model. This could perhaps be improved through a porosity dependant diffusion coefficient or by deriving an averaged expression for the forces or stresses near close packing. Improving this aspect should be the priority in future works, but most of the aforementioned changes should be made for the model to become viable for dense particulate flows. The VANS LBM could also be applied to other multiphase and multicomponent flows and could easily be combined with discrete element methods or potentially be adapted to develop a new two-fluid LBM.

\section{Appendix A. Chapman-Enskog analysis of the novel scheme}

This section provides an analysis of the novel scheme. The notations and procedure will be analogous to that of Blais et al [12]. The aim is to investigate the behaviour of the scheme in the hydrodynamic limit and to determine their relation to the VANS equations, as given by (2) and (3)

$$
\begin{gathered}
\frac{\partial}{\partial t}(\epsilon \rho)+\nabla \cdot(\epsilon \rho\langle\mathbf{u}\rangle)=0 \\
\frac{\partial}{\partial t} \epsilon \rho\langle\mathbf{u}\rangle+\nabla \cdot\left(\epsilon \rho\langle\mathbf{u}\rangle\left\langle\mathbf{u}^{\top}\right\rangle\right)+\epsilon \nabla\langle p\rangle=\mu \Delta(\epsilon\langle\mathbf{u}\rangle)+\mathbf{F}^{h} .
\end{gathered}
$$

For this result we use the adapted lattice Boltzmann Eq. (8) from Section 3.2

$$
\begin{aligned}
f_{i}(\mathbf{x} & \left.+\xi_{i} \delta t, t+\delta t\right)-f_{i}(\mathbf{x}, t) \\
& =\frac{1}{\tau}\left(f_{i}^{e q}-f_{i}\right)+\left(\epsilon\left(\mathbf{x}+\boldsymbol{\xi}_{i} \delta t, t+\delta t\right)-\epsilon(\mathbf{x}, t)\right) \frac{w_{i} \sum_{j} f_{j}(\mathbf{x}, t)}{\epsilon(\mathbf{x}, t)},
\end{aligned}
$$

as well as the equilibrium function

$f_{i}^{e q}=\epsilon \rho w_{i}\left(1+\frac{\boldsymbol{\xi}_{i} \cdot\langle\mathbf{u}\rangle}{c_{s}^{2}}+\frac{\left(\boldsymbol{\xi}_{i} \cdot\langle\mathbf{u}\rangle\right)^{2}}{2 c_{s}^{4}}-\frac{\langle\mathbf{u}\rangle \cdot\langle\mathbf{u}\rangle}{2 c_{s}^{2}}\right)$.

For simplicity's sake the averaging operator $\langle\cdot\rangle$ is omitted in the following derivation.

The moments of the equilibrium function are given by

$$
\begin{aligned}
\sum_{i} f_{i}^{e q} & =\epsilon \rho \\
j_{\alpha}^{e q} & :=\sum_{i} f_{i}^{e q} \xi_{i \alpha}=\epsilon \rho u_{\alpha} \\
\Pi_{\alpha \beta}^{e q} & :=\sum_{i} f_{i}^{e q} \xi_{i \alpha} \xi_{i \beta}=c_{s}^{2} \epsilon \rho \delta_{\alpha \beta}+\epsilon \rho u_{\alpha} u_{\beta} \\
S_{\alpha \beta \gamma}^{e q} & :=\sum_{i} f_{i}^{e q} \xi_{i \alpha} \xi_{i \beta} \xi_{i \gamma}=c_{s}^{2} \epsilon \rho\left(u_{\alpha} \delta_{\beta \gamma}+u_{\beta} \delta_{\alpha \gamma}+u_{\gamma} \delta_{\alpha \beta}\right) .
\end{aligned}
$$

With the use of an expansion parameter $\lambda$ the different scales are separated, yielding

$$
\begin{aligned}
& f_{i}=f_{i}^{(0)}+\lambda f_{i}^{(1)}+\lambda^{2} f_{i}^{(2)}+\mathcal{O}\left(\lambda^{3}\right), \\
& \partial_{t}=\lambda \partial_{t}^{(1)}+\lambda^{2} \partial_{t}^{(2)}, \quad \nabla=\lambda \nabla^{(1)} .
\end{aligned}
$$

Inserting this into Eq. (A.1) and sorting the different powers of $\lambda$ gives

$$
\begin{aligned}
& \lambda^{0}: 0=-\frac{1}{\tau}\left(f_{i}^{e q}-f_{i}^{(0)}\right) \quad \Leftrightarrow \quad f_{i}^{e q}=f_{i}^{(0)} \\
& \lambda^{1}:\left(\partial_{t}^{(1)}+\xi_{i} \nabla^{(1)}\right) f_{i}^{(0)}=-\frac{\omega}{\delta t} f_{i}^{(1)}+w_{i} \frac{\sum_{j} f_{j}^{(0)}}{\epsilon}\left(\partial_{t}^{(1)}+\xi_{i} \nabla^{(1)}\right) \epsilon .
\end{aligned}
$$

By applying the derivative $\partial_{t}^{(1)}+\xi_{i} \cdot \nabla^{(1)}$ to Eq. (A.2) this results in

$$
\begin{aligned}
\frac{\omega}{\delta t}\left(\partial_{t}^{(1)}+\boldsymbol{\xi}_{i} \cdot \nabla^{(1)}\right) f_{i}^{(1)}= & \left(\partial_{t}^{(1)}+\boldsymbol{\xi}_{i} \cdot \nabla^{(1)}\right)^{2} f_{i}^{(0)} \\
& +\frac{w_{i} \sum_{j} f_{j}^{(0)}}{\epsilon}\left(\partial_{t}^{(1)}+\boldsymbol{\xi}_{i} \cdot \nabla^{(1)}\right)^{2} \epsilon
\end{aligned}
$$

where the derivative of $\frac{w_{i} \sum_{j} f_{j}^{(0)}}{\epsilon}$ has been neglected, which is valid if pressure fluctuations remain relatively small whenever there are large porosity gradients. One could furthermore argue, that this is correct since $\pi_{i}$ and $v_{i}$ are streamed independently instead of determined anew after the streaming step.

Combining (A.3) with the terms of magnitude $\lambda^{2}$ gives:

$$
\begin{gathered}
\lambda^{2}: \partial_{t}^{(2)} f_{i}^{(1)}+\left(1-\frac{\omega}{2}\right)\left(\partial_{t}^{(1)}+\xi_{i} \cdot \nabla^{(1)}\right) f_{i}^{(1)}=-\frac{\omega}{\delta_{t}} f_{i}^{(2)} \\
+\frac{w_{i} \sum_{j} f_{j}^{(0)}}{\epsilon} \partial_{t}^{(2)} \epsilon+\frac{w_{i} \sum_{j} f_{j}^{(1)}}{\epsilon}\left(\partial_{t}^{(1)}+\xi_{i} \cdot \nabla^{(1)}\right) \epsilon .
\end{gathered}
$$

Taking the moments of Eqs. (A.2) and (A.4), while using that $0=$ $\sum_{i} f_{i}^{(1)}=\sum_{i} f_{i}^{(2)}=\sum_{i} \xi_{i} f_{i}^{(1)}$ and $0=\sum_{i} w_{i} \xi_{i} \sum_{j} f_{j}^{(0)}$ due to symmetry, as well as $\epsilon \rho c_{s}^{2} \mathbb{I}=\sum_{i} w_{i} \xi_{i} \xi_{i} \sum_{j} f_{j}^{(0)}$, results in

$\sum_{i} \lambda: \quad \partial_{t}^{(1)}(\epsilon \rho)+\nabla^{(1)} \cdot(\epsilon \rho \mathbf{u})=\rho \partial_{t}^{(1)} \epsilon$,

$\sum_{i} \lambda^{2}: \quad \partial_{t}^{(2)}(\epsilon \rho)=\rho \partial_{t}^{(2)} \epsilon$ 
$\sum_{i} \xi_{i} \lambda: \quad \partial_{t}^{(1)}(\epsilon \rho \mathbf{u})+\nabla^{(1)} \cdot\left(\epsilon \rho c_{s}^{2} \mathbb{I}+\epsilon \rho \mathbf{u u}^{\top}\right)=\rho c_{s}^{2} \mathbb{I} \cdot \nabla^{(1)} \epsilon$,

$\sum_{i} \xi_{i} \lambda^{2}: \quad \partial_{t}^{(2)}(\epsilon \rho \mathbf{u})+\left(1-\frac{\omega}{2}\right) \nabla^{(1)} \cdot \Pi^{(1)}=0$.

The corresponding macroscopic equations are given by

$\sum_{i} \lambda+\lambda^{2}: \quad \epsilon \partial_{t} \rho+\nabla \cdot(\epsilon \rho \mathbf{u})=0$,

$\sum_{i} \xi\left(\lambda+\lambda^{2}\right): \quad \partial_{t}(\epsilon \rho \mathbf{u})+\nabla \cdot\left(\epsilon \rho \mathbf{u u}^{\top}\right)+\epsilon \nabla p$

$=\left(1-\frac{\omega}{2}\right) \nabla \cdot \Pi^{(1)}$.

$\Pi^{(1)}$ can be derived through the third moment of Eq. (A.2)

$\sum_{i} \xi_{i} \xi_{i} \lambda: \quad \delta_{t}^{(1)} \boldsymbol{\Pi}^{(0)}+\nabla^{(1)} \cdot S^{(0)}=\frac{\omega}{\delta t} \boldsymbol{\Pi}^{(1)}+\rho c_{s}^{2} \mathbb{I} \partial_{t}^{(1)} \epsilon$.

In order to derive these quantities Einstein notation is used. In order to simplify $\left(\partial_{t}^{(1)} \Pi^{(0)}-\rho c_{s}^{2} \mathbb{I} \partial_{t}^{(1)} \epsilon\right)_{\alpha \beta}=\epsilon \partial_{t}^{(1)}\left(\rho \delta_{\alpha \beta} c_{s}^{2}\right)+$ $\partial_{t}^{(1)}\left(\rho \in u_{\alpha} u_{\beta}\right)$, (A.5) and (A.6) are used, which gives

$$
\begin{aligned}
\epsilon \partial_{t}^{(1)} \rho & =-\left(\epsilon \rho u_{\alpha}\right)_{, \alpha} \\
\partial_{t}^{(1)}\left(\epsilon \rho u_{\alpha}\right) & =-\epsilon\left(\rho \delta_{\alpha \beta} c_{s}^{2}\right)_{\beta}-\left(\epsilon \rho u_{\alpha} u_{\beta}\right),_{\beta} .
\end{aligned}
$$

Accordingly,

$$
\begin{aligned}
\epsilon \delta_{t}^{(1)} & \left(\rho \delta_{\alpha \beta} c_{s}^{2}\right)+\partial_{t}^{(1)}\left(\rho \epsilon u_{\alpha} u_{\beta}\right) \\
= & \epsilon \delta_{t}^{(1)}\left(\rho \delta_{\alpha \beta} c_{s}^{2}\right)+u_{\alpha} \partial_{t}^{(1)}\left(\rho \epsilon u_{\beta}\right)+u_{\beta} \partial_{t}^{(1)}\left(\rho \epsilon u_{\alpha}\right) \\
& +u_{\alpha} u_{\beta} \partial_{t}^{(1)}(\rho \epsilon) \\
= & -\left(\epsilon \rho u_{\gamma}\right)_{\gamma} \delta_{\alpha \beta} c_{s}^{2}-u_{\alpha}\left(\epsilon\left(\rho c_{s}^{2}\right),_{\beta}-\left(\epsilon \rho u_{\beta} u_{\gamma}\right),_{\gamma}\right) \\
& -u_{\beta}\left(\epsilon\left(\rho c_{s}^{2}\right)_{\alpha}-\left(\epsilon \rho u_{\alpha} u_{\gamma}\right)_{\gamma}\right)+u_{\alpha} u_{\beta} \partial_{t}^{(1)}(\rho \epsilon) .
\end{aligned}
$$

For $\nabla^{(1)} \cdot S^{(0)}$ one obtains

$$
\begin{aligned}
& \left(c_{s}^{2} \epsilon \rho\left(u_{\alpha} \delta_{\beta \gamma}+u_{\beta} \delta_{\alpha \gamma}+u_{\gamma} \delta_{\alpha \beta}\right)\right), \gamma \\
& \quad=c_{s}^{2}\left(\epsilon \rho u_{\alpha}\right)_{\beta}+c_{s}^{2}\left(\epsilon \rho u_{\beta}\right)_{\alpha}+c_{s}^{2} \delta_{\alpha \beta}\left(\epsilon \rho u_{\gamma}\right), \gamma .
\end{aligned}
$$

Inserting the results from Eqs. (A.8) and (A.9) into Eq. (A.7) while eliminating $\mathrm{M}^{3}$ terms, where $\mathrm{M}$ is the Mach number, gives

$$
\begin{aligned}
\Pi_{\alpha \beta}^{(1)}= & \delta t \tau\left(-\epsilon u_{\alpha}\left(\rho c_{s}^{2}\right), \beta-\epsilon u_{\beta}\left(\rho c_{s}^{2}\right)_{, \alpha}\right. \\
& +\left(\epsilon \rho c_{s}^{2} u_{\alpha}\right)_{\beta}+\left(\epsilon \rho c_{s}^{2} u_{\beta}\right)_{,} \\
= & -c_{s}^{2} \tau \delta t\left(\rho\left(\epsilon u_{\alpha}\right)_{, \beta}+\rho\left(\epsilon u_{\beta}\right)_{, \alpha}\right) .
\end{aligned}
$$

Inserting this into the conservation equation yields

$$
\begin{aligned}
& \partial_{t}\left(\epsilon \rho u_{\beta}\right)+\left(\epsilon \rho u_{\alpha} u_{\beta}\right)_{, \alpha}+\epsilon \cdot\left(\rho c_{s}^{2} \delta_{\alpha \beta}\right)_{, \alpha} \\
& \quad=-c_{s}^{2}\left(1-\frac{\omega}{2}\right) \delta t \tau\left(\rho\left(\epsilon u_{\alpha}\right)_{, \beta}+\rho\left(\epsilon u_{\beta}\right)_{, \alpha}\right)_{, \beta} .
\end{aligned}
$$

Substituting $\langle p\rangle=\rho c_{s}^{2}$ and $v=\left(\tau-\frac{1}{2}\right) c_{S}^{2} \delta t$ results in the macroscopic equations

$$
\begin{aligned}
& \epsilon \partial_{t} \rho+\nabla \cdot(\epsilon \rho\langle\mathbf{u}\rangle)=0 \\
& \partial_{t} \epsilon \rho\langle\mathbf{u}\rangle+\nabla \cdot\left(\epsilon \rho\langle\mathbf{u}\rangle\langle\mathbf{u}\rangle^{\top}\right)+\epsilon \nabla\langle p\rangle \\
& \quad=v \nabla \cdot\left(\rho \nabla(\epsilon\langle\mathbf{u}\rangle)+\rho \nabla(\epsilon\langle\mathbf{u}\rangle)^{\top}\right) .
\end{aligned}
$$

\section{References}

[1] Tien C, Payatakes AC. Advances in deep bed filtration. AlChE J 1979;25(5):73759. doi:10.1002/aic.690250502.

[2] McDowellBoyer LM, Hunt JR, Sitar N. Particle transport through porous media. Water Resour Res 1986;22(13):1901-21. doi:10.1029/WR022i013p01901.

[3] Xu BH, Yu AB. Numerical simulation of the gas-solid flow in a fluidized bed by combining discrete particle method with computational fluid dynamics. Chem Eng Sci 1997;52(16):2785-809. doi:10.1016/S0009-2509(97)00081-X.

[4] Ishii M, Hibiki T. Thermo-fluid dynamics of two-Phase flow. Springer New York; 2011. ISBN 9781441979858.

[5] Maier M-L, Henn T, Thter G, Nirschl H, Krause MJ. Multiscale simulation with a two-way coupled lattice Boltzmann method and discrete element method. Chem Eng Technol 2017:40(9):1591-8. doi:10.1002/ceat.201600547.

[6] Henn T, Thäter G, Dörfler W, Nirschl H, Krause MJ. Parallel dilute particulate flow simulations in the human nasal cavity. Comput Fluids 2016;124:197-207. doi:10.1016/j.compfluid.2015.08.002.

[7] Anderson TB, Jackson R. Fluid mechanical description of fluidized beds equations of motion. Ind Eng Chem Fundam 1967;6(4):527-39. doi:10.1021/ i160024a007.

[8] Enwald H, Peirano E, Almstedt AE. Eulerian two-phase flow theory applied to fluidization. Int J Multiphase Flow 1996;22(Suppl. 1):21-66.

[9] Jackson R. Locally averaged equations of motion for a mixture of identical spherical particles and a newtonian fluid. Chem Eng Sci 1997;52(15):2457-69. doi:10.1016/S0009-2509(97)00065-1.

[10] Nithiarasu P, Seetharamu KN, Sundararajan T. Natural convective heat transfer in a fluid saturated variable porosity medium. Int J Heat Mass Transf 1997;40(16):3955-67.

[11] Zhou ZY, Kuang SB, Chu KW, Yu AB. Discrete particle simulation of particle-fluid flow: model formulations and their applicability. J Fluid Mech 2010;661:482-510. doi:10.1017/S002211201000306X.

[12] Blais B, Tucny JM, Vidal D, Bertrand F. A conservative lattice Boltzmann model for the volume-averaged Navier-Stokes equations based on a novel collision operator. J Comput Phys 2015;294:258-73. doi:10.1016/j.jcp.2015.03.036.

[13] Chen S, Doolen GD. Lattice Boltzmann method for fluid flows. Annu Rev Fluid Mech 1998;30(1):329-64.

[14] Guo Z, Shu C. Lattice boltzmann method and its applications in engineering. World Scientific; 2013. doi:10.1142/9789814508308_0001.

[15] Krause MJ, Gengenbach T, Heuveline V. Hybrid parallel simulations of fluid flows in complex geometries: application to the human lungs. In: Guarracino $M$, Vivien $F$, Traeff J, Cannatoro $M$, Danelutto $M$, Hast A, Perla F, Knuepfer A, Di Martino B, Alexander M, editors. Euro-Par 2010 parallel processing workshops. Lecture Notes in Computer Science, 6586. Springer Berlin / Heidelberg; 2011. p. 209-16.

[16] Heuveline V, Krause MJ. OpenLB: towards an efficient parallel open source library for lattice Boltzmann fluid flow simulations. PARA'08 workshop on state-of-the-art in scientific and parallel computing, May 13-16, 2008 No 6126 , 6127. Springer series Lecture Notes in Computer Science (LNCS); 2011.

[17] Guo Z, Zhao TS. Lattice Boltzmann model for incompressible flows through porous media. Phys Rev E - Stat Nonlinear Soft Matter Phys 2002;66(3) 036304/1-036304/9. doi:10.1103/PhysRevE.66.036304.

[18] Zhang J, Wang L, Ouyang J. Lattice Boltzmann model for the volume-averaged Navier-Stokes equations. EPL 2014;107(2). doi:10.1209/0295-5075/107/20001.

[19] Song F, Wang W, Li J. A lattice Boltzmann method for particle-fluid two-phase flow. Chem Eng Sci 2013;102:442-50. doi:10.1016/j.ces.2013.08.037.

[20] Guo Z, Zheng C, Shi B. Discrete lattice effects on the forcing term in the lattice Boltzmann method. Phys Rev E - Stat NonlinearSoft Matter Phys 2002;65(4) 046308/1-046308/6. doi:10.1103/PhysRevE.65.046308.

[21] Whitaker S. The method of volume averaging. Theory and applications of transport in porous media. Dordrecht: Kluwer; 1999. ISBN 0-7923-5486-9.

[22] Crowe CT, editor. Multiphase flows with droplets and particles. 2nd. Boca Raton, Fla.: CRC Press; 2012. ISBN 978-1-4398-4050-4.

[23] Junk M, Klar A, Luo L-S. Asymptotic analysis of the lattice Boltzmann equation. J Comput Phys 2005;210(2):676-704. doi:10.1016/j.jcp.2005.05.003.

[24] Wolf-Gladrow D. Lattice-gas cellular automata and lattice Boltzmann models an introduction, 308. Berlin: Springer; 2000.

[25] Trunk R, Henn T, Dņrfler W, Nirschl H, Krause MJ. Inertial dilute particulate fluid flow simulations with an Euler-Euler lattice Boltzmann method. J Comput Sci 2016;17(Part 2):438-45. Discrete Simulation of Fluid Dynamics 2015. doi: 10.1016/j.jocs.2016.03.013.

[26] Zhang H, Yuan H, Trias FX, Yu A, Tan Y, Oliva A. Particulate immersed boundary method for complex fluid-particle interaction problems with heat transfer. Comput Math Appl 2016;71(1):391-407. doi:10.1016/j.camwa.2015.12.003. 
Karlsruher Institut für Technologie

\section{Repository KITopen}

Dies ist ein Postprint/begutachtetes Manuskript.

Empfohlene Zitierung:

Höcker, S. B.; Trunk, R.; Dörfler, W.; Krause, M. J.

Towards the simulations of inertial dense particulate flows with a volume-averaged lattice Boltzmann method.

2018. Computers \& fluids, 166.

doi: $\underline{10.5445 / I R / 1000080937}$

Zitierung der Originalveröffentlichung:

Höcker, S. B.; Trunk, R.; Dörfler, W.; Krause, M. J.

Towards the simulations of inertial dense particulate flows with a volume-averaged lattice Boltzmann method.

2018. Computers \& fluids, 166, 152-162.

doi:10.1016/j.compfluid.2018.02.011

Lizenzinformationen: CC BY-NC-ND 4.0 